apuntesuniversitarios.upeu.edu.pe

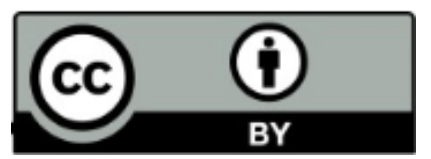

Apuntes Universitarios, 2020: 10(1), Enero-Marzo

ISSN: 2304-0335 DOI: https://doi.org/10.17162/au.v10i1.415

\title{
Actitudes hacia la estadística en ciencias económico-administrativas: un análisis por etapa de pregrado
}

\section{Attitudes towards statistics in economic and administrative sciences: An analysis by undergraduate stage}

\author{
Carlos Gerardo Torres Ceballos ${ }^{1 \mathrm{a}}$
}

Universidad de Guadalajara, Centro Universitario de Ciencias Económico Administrativas ${ }^{1}$

Orcid ID: https://orcid.org/0000-0002-4059-1736 ${ }^{1}$

Recibido: 11 de junio 2019

Aceptado: 11 de setiembre 2019

\section{Resumen}

La presente investigación tuvo por objetivos: 1) caracterizar las actitudes hacia la estadística en las etapas iniciales, intermedias y finales de pregrados en Ciencias Económico Administrativas y, 2) determinar las aplicaciones que los alumnos dan a la estadística durante sus estudios universitarios. Participaron 159 estudiantes (69.18\% mujeres y 30.81\% varones) de carreras como Recursos Humanos, Administración, Negocios Internacionales y Mercadotecnia, los cuales contestaron un cuestionario de diferencial semántico integrado por siete pares de adjetivos extremos con base en una escala de $-3 \mathrm{a}+3$. Los resultados mostraron que, durante las tres etapas de las carreras (inicial, intermedia y final), las actitudes hacia la estadística se mantienen algo positivas (con promedios de $0.73,0.94$ y 1.00 , respectivamente) y que, aunque se percibe su importancia, son algo tensas para los estudiantes. Asimismo, se encontró que dichas actitudes son utilizadas para comprender información, calcular datos e investigar. Además, se discuten posibles explicaciones para tales hallazgos, por ejemplo, las estrategias didácticas de los docentes y la historia académica de los estudiantes.

Palabras clave: actitudes, estadística, pregrado, utilidad, Ciencias Económico-Administrativas.

aCorrespondencia al autor

E-mail: carlos.torres@cucea.udg.mx 


\begin{abstract}
The purpose of this research was to: 1) characterize attitudes towards statistics in the initial, intermediate and final stages of undergraduate degrees in Administrative Economic Sciences and, 2) determine the applications that students give to statistics during their university studies. 159 students (69.18\% female and 30.81\% male) participated in careers such as Human Resources, Administration, International Business and Marketing, which answered a semantic differential questionnaire composed of seven pairs of extreme adjectives based on a scale of -3 to +3 . The results showed that, during the three stages of the races (initial, intermediate and final), the attitudes towards statistics remain somewhat positive (with averages of $0.73,0.94$ and 1.00, respectively) and that, although their importance is perceived, they are somewhat tense for students. It was also found that these attitudes are used to understand information, calculate data and investigate. In addition, possible explanations for such findings are discussed, for example, the teaching strategies of teachers and the academic history of students.
\end{abstract}

Keywords: attitudes, statistics, undergraduate, utility, Economic-Administrative Sciences.

\title{
Introducción
}

Como ciencia matemática, la estadística sintetiza datos cuantitativos de individuos, grupos y eventos y establece conjeturas para tomar decisiones (Barreto, 2012). En el nivel global, permite a los gobiernos, organizaciones e instituciones solucionar problemas sociales (Cuadras, 2006). En las empresas, se utiliza para planear, presupuestar, controlar procesos, evaluar el desempeño, gestionar personal, elaborar nóminas, auditar, etc. (Jiménez, Ocegueda, García \& Guzmán, 2013; Liquidano, 2009; Pasten, Montiel \& Torres, 2010).

En la universidad, la asignatura respectiva promueve en los estudiantes el pensamiento crítico, la conexión de ideas, la lógica y la comprensión y explicación de fenómenos (Rouquette, Suárez \& Ariza, 2014). Asimismo, es indispensable para desarrollar investigación en todas las áreas del conocimiento científico (Gil, 2003; Osorio \& Suárez, 2014). En el nivel individual, la estadística permite reconocer y entender la incertidumbre y el azar cotidiano (Eudave, 2007).

No obstante, pese a la importancia de la materia, entre un $37 \%$ y $68 \%$ de los universitarios mexicanos la reprueban, aunque sea de tronco común en la mayoría de los programas de pregrado (Alonso, 2013; Aguilar, Lara, Iñiguez \& Rivera, 2018; Corona, Reyes, Martínez \& Rivas, 2016; Robles, Cisneros \& Guzmán, 2018). Una de las explicaciones de tal reprobación hacia la estadística, se pueden asociar con el aprendizaje deficiente de la materia, bajas calificaciones, estrés y ansiedad (Sesé, Jiménez, Montaño \& Palmer, 2015), las cuales disminuyen el rendimiento y el interés hacia esta ciencia (Pérez, Aparicio, Bazán \& Abdounur, 2015). Por el contrario, las 
actitudes positivas favorecen los procesos educativos, el desempeño y las buenas calificaciones (Bautista, Morales, Dórame \& Peralta, 2016; Rosli \& Maat, 2017).

Por otro lado, las actitudes hacia la estadística varían por área de conocimiento. Verbigracia, los estudiantes de Humanidades (Pedagogía, Psicología, Ciencias Sociales, etc) cuentan con actitudes indiferentes o negativas (Landa, 2015; Vilá \& Rubio, 2016) y los de ciencias exactas (Ingeniería, Matemáticas, Computación, etc) y ciencias de la salud (Medicina, Enfermería, etc.) tienen actitudes más positivas (Páez, Burne, Mosconi \& Montenegro, 2017; Peña et al., 2015; Salazar \& Acón, 2017; Stanisavljevic et al., 2014).

Para los estudiantes de Ciencias económico administrativas (Recursos Humanos, Administración, Mercadotecnia, Negocios Internacionales, etc.) los datos no son concluyentes (Díaz, 2012). Por ejemplo, se han reportado actitudes de indiferencia (Coetzee \& Merwe, 2010) pero también actitudes relativamente favorables (Acosta \& Mejía, 2017; Bautista, Morales, Dórame \& Peralta, 2016).

De igual manera, existen diferencias por área respecto a las creencias sobre la utilidad de la estadística para el ejercicio profesional (Ashaari, Judi, Mohamed \& Wook, 2011). Por ejemplo, en el campo de las Humanidades la estadística se considera poco útil, dificil e incluso aversiva (Escalante, Repetto \& Mattinello, 2011; Ruiz, 2015); en ciencias exactas y ciencias de la salud se considera útil, fácil y agradable (Santabárbara \& López, 2019). Sin embargo, en Ciencias económico administrativas se considera útil pero difícil y aversiva a la vez (Pérez, Aparicio, Bazán \& Abdounur, 2015).

Estas creencias contradictorias en el área económico administrativa se podrían clarificar analizando información de distintas etapas de pregrado, para detectar si conforme se va usando la estadística las actitudes hacia ella se vuelven más favorables. Sin embargo, la mayoría de las investigaciones reportan datos de una sola etapa de licenciatura. Incluso, no hay suficiente información sobre los usos de la estadística por parte de los universitarios.

Entonces, el presente estudio tuvo por objetivo: 1) caracterizar las actitudes hacia la estadística (en estudiantes de carreras económico administrativas) en diferentes etapas de pregrado (inicial, intermedia y final); y 2) conocer las opiniones de los alumnos respecto a la utilidad de dicha disciplina. Además, se esperaba encontrar que las actitudes fueran más positivas durante la etapa final que en la etapa inicial. En el menor de los casos, se esperaba encontrar que los estudiantes usaran la estadística para sus tareas durante el pregrado. 


\section{Método}

\section{Tipo de estudio}

El paradigma del presente estudio fue cuantitativo, pues consideró la medición objetiva de las actitudes. El método fue no experimental (pues no se manipularon variables), de tipo descriptivo (se describió la situación prevaleciente al momento de realizarse el estudio) y de sección transversal (examinó a varios grupos de personas en un mismo punto del tiempo) (Salkind, 1999).

\section{Población y muestra}

La población se compuso por estudiantes regulares de pregrado, siendo alumnos presenciales del área económico administrativa, vinculados a la gestión empresarial. Sus clases se impartieron en una universidad pública de Jalisco, México. El muestreo fue no probabilístico por conveniencia, ya que se aplicaron los cuestionarios de acuerdo con la disponibilidad de horarios de clase (Salkind, 1999). Las características de la muestra se exponen en la Tabla 1.

Tabla 1

Características de la muestra de estudiantes $(n=159)$

\begin{tabular}{llr}
\hline Pregrado & \multicolumn{1}{c}{$\%$} \\
& Recursos Humanos & 39.62 \\
& Administración & 35.22 \\
& Negocios Internacionales & 16.35 \\
Etapa de pregrado & Mercadotecnia & 8.81 \\
& & \\
& Inicial (semestre 1 al 3) & 16.98 \\
Sexo & Intermedia (semestre 4 al 6) & 47.79 \\
& Final (semestre 7 al 9) & 35.23 \\
Edad en años & Mujeres & 69.18 \\
& Hombres & 30.82 \\
& & 35.22 \\
& $18-20$ & 51.57 \\
& $21-23$ & 13.21 \\
\hline
\end{tabular}




\section{Instrumento}

Se utilizó la técnica de Semántica Diferencial de Osgood, una de las técnicas más utilizadas para la medición de las actitudes y se ha demostrado su validez y confiabilidad en diferentes estudios (Arce, Andrade \& Seoane, 1997). Esta consiste en construir una escala bipolar tipo diferencial semántico, estableciendo una serie de adjetivos extremos, a fin de caracterizar las percepciones de los alumnos y calificar al objeto de actitud (Pérez, Arango \& Branch, 2008), en este caso la estadística.

El instrumento constó de una primera sección para recabar datos generales y el consentimiento informado de los estudiantes. La segunda sección consistió en siete pares de adjetivos extremos con una escala del -3 a +3 (ver Figura 2). La tercera sección consistió en una pregunta abierta respecto a la utilidad de la estadística. El cuestionario podía ser contestado en 10 minutos o menos.

\section{Procedimiento}

De manera previa a la aplicación, se llevó a cabo un piloteo del instrumento a fin de evaluar aspectos de claridad en la redacción y decidir los pares de adjetivos bipolares extremos pertinentes. La versión revisada del instrumento se aplicó de manera colectiva en las aulas de la universidad durante el semestre 2018A. Los aplicadores señalaron verbalmente a los estudiantes que el estudio era únicamente para conocer su opinión sobre la estadística y que no tenía ninguna influencia sobre sus calificaciones.

\section{Resultados}

Los datos recolectados con el diferencial semántico se procesaron con el programa Excel. Para el análisis, los alumnos fueron divididos en tres grupos de acuerdo con la etapa de pregrado que se encontraban cursando: a) inicial, si se encontraban en primero, segundo o tercer semestre; b) intermedio, si se encontraban en cuarto, quinto o sexto semestre y; c) final, si se encontraban en séptimo, octavo o noveno semestre.

En primer lugar, se obtuvo el promedio general y la desviación típica del puntaje total del cuestionario por cada grupo para caracterizar sus actitudes como negativas, indiferentes o positivas en función de la ubicación del promedio dentro de la escala utilizada. Los resultados se muestran en la Figura 1. 


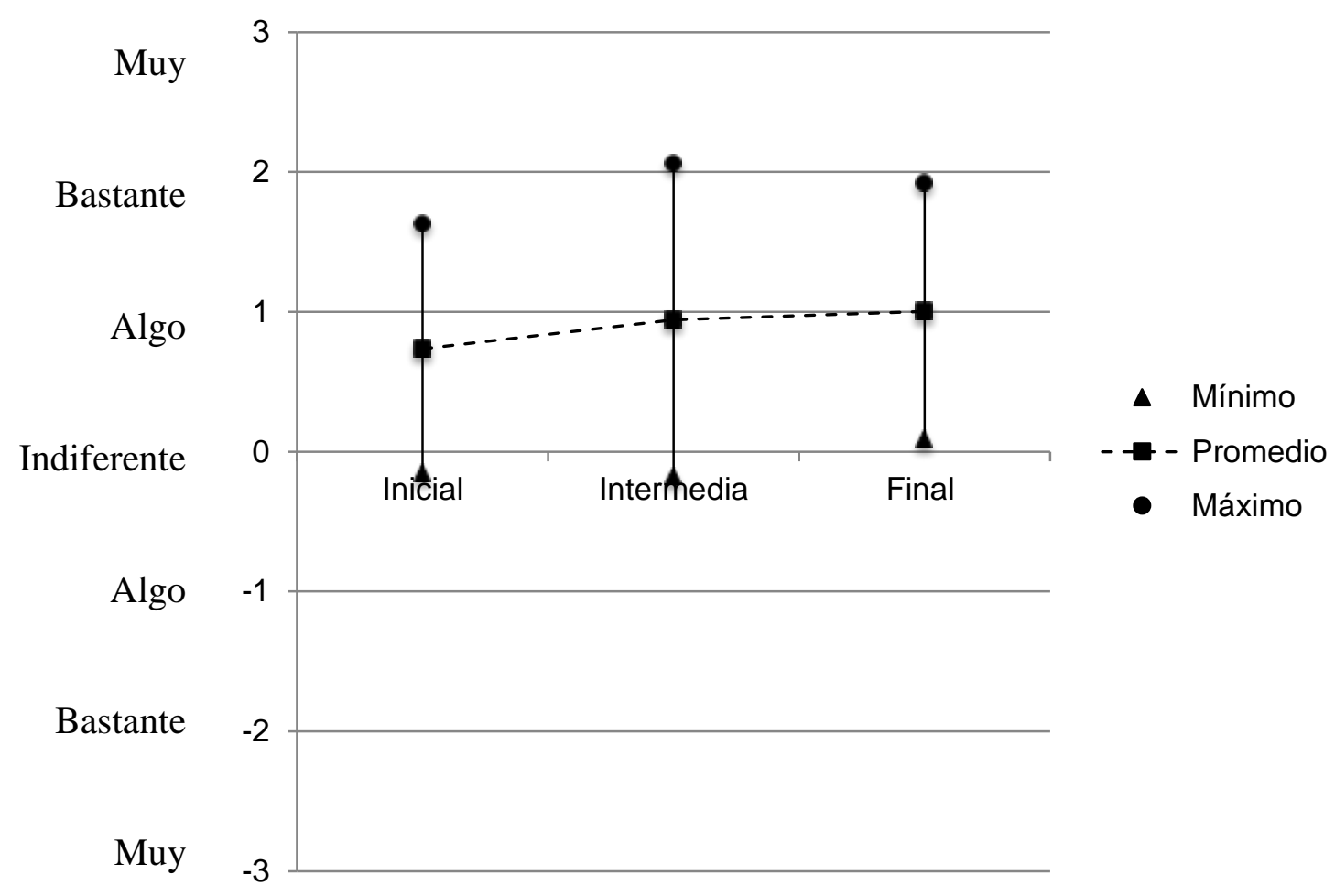

Figura 1. Promedio y desviación típica de las actitudes hacia la estadística por etapa de pregrado.

En la Figura 1 se puede observar que las actitudes hacia la estadística se mantienen algo positivas durante todo el pregrado. Cabe señalar que los puntajes negativos del límite inferior tienden a mejorar en la etapa final hasta alcanzar la indiferencia. Posteriormente, se obtuvo el promedio por grupo para cada uno de los siete pares de adjetivos bipolares extremos. Los resultados se presentan en la siguiente Figura 2. 


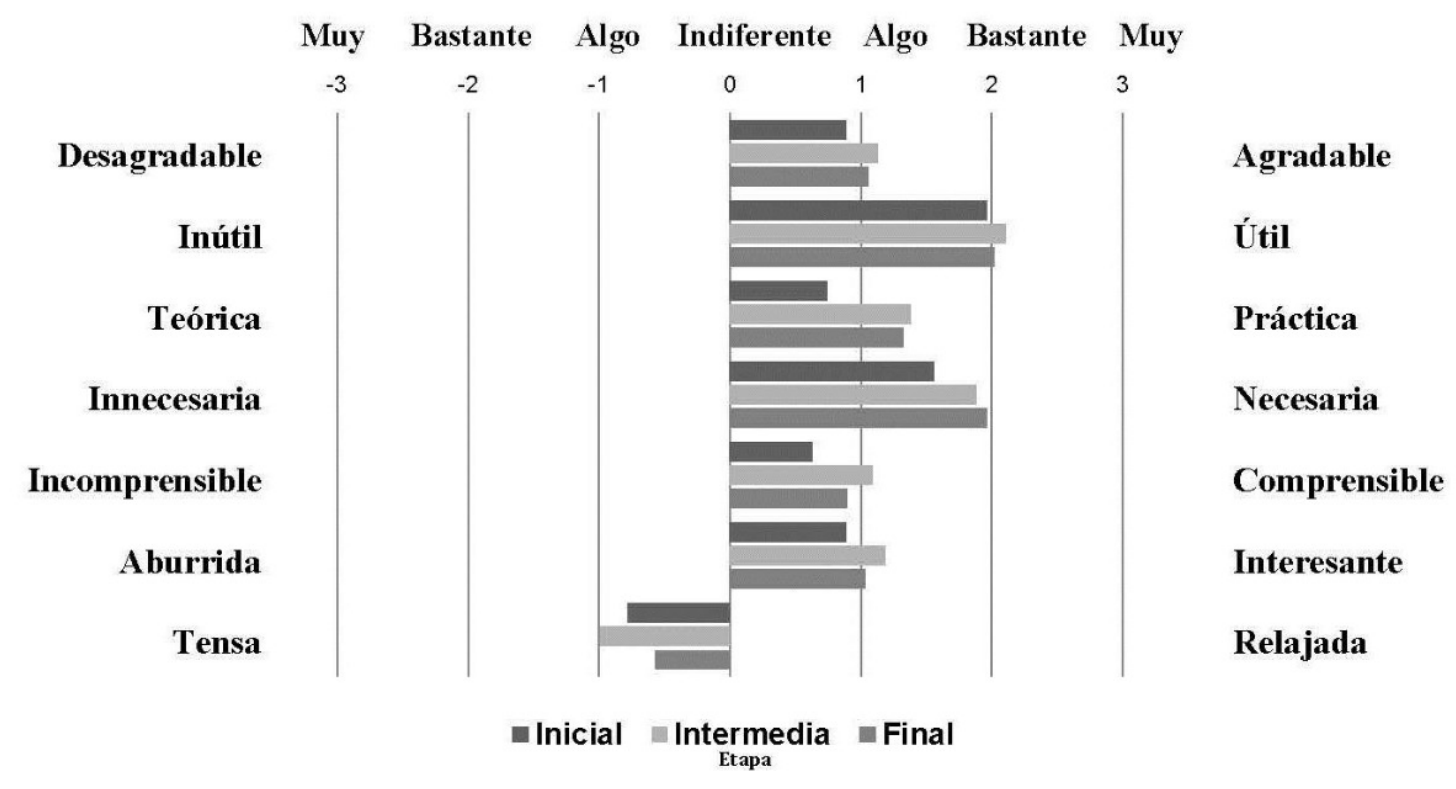

Figura 2. Puntaje promedio de cada adjetivo bipolar extremo por etapa de pregrado.

En la Figura 2 se puede corroborar que la mayoría de las creencias hacia la estadística tienden a mantenerse estables durante el pregrado. Por ejemplo, durante las tres etapas se le considera algo agradable, bastante útil, algo comprensible, algo interesante y algo tensa. Sin embargo, otras creencias cambian moderadamente entre etapas. Por ejemplo, tiende a considerarse más práctica y más necesaria conforme se avanza en la carrera. Posteriormente, se preguntó a los alumnos sobre la utilidad de la estadística mediante un ítem de respuesta abierta. Las respuestas se ordenaron en las categorías que se muestran en la siguiente Figura 3. 


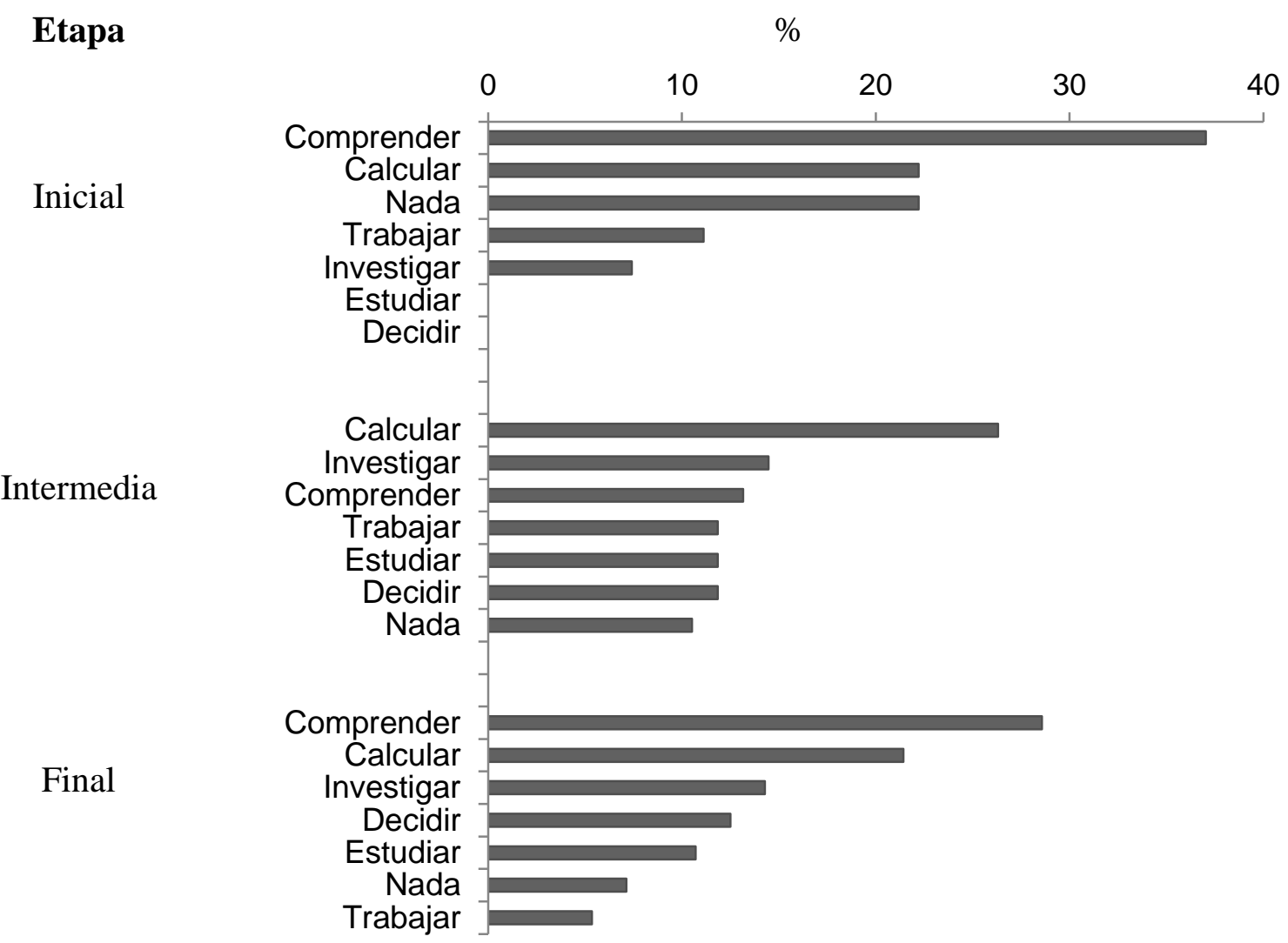

Figura 3. Categorías de respuesta para la pregunta ¿De qué te ha servido la estadística? por etapa de pregrado.

En la Figura 3 se puede observar que, durante la etapa inicial y durante la etapa final de pregrado, la estadística se usa principalmente para comprender información y luego para calcular datos. En la etapa intermedia se usa primordialmente para calcular datos y después para realizar investigación. De igual manera, se puede observar que el porcentaje de alumnos que considera que la estadística no le ha servido disminuye después de la primera etapa. Del mismo modo, el porcentaje de alumnos que reporta utilizar la estadística como herramienta para la toma de decisiones aumenta en los últimos semestres del pregrado. 


\section{Discusión}

Este estudio busca determinar las actitudes hacia la estadística en estudiantes de Ciencias Económico Administrativas en diferentes etapas de pregrado. En general, resultaron algo positivas durante toda la carrera lo cual coincide con otros trabajos (Acosta \& Mejía, 2017; Bautista, Morales, Dórame \& Peralta, 2016; Coetzee \& Van der Merwe, 2010; Griffith et al., 2012) que señalan que las actitudes en esta población oscilan entre indiferentes y moderadamente positivas. Asimismo, coinciden con otros estudios que han encontrado que esta población considera a la estadística como bastante útil y algo comprensible; y a la vez algo tensa (Pérez, Aparicio, Bazán \& Abdounur, 2015).

Una explicación de que las actitudes sean tensas o estresantes para la muestra estudiada podría ser la necesidad de estrategias didácticas para asociar la estadística con estímulos agradables (Barreto, 2012; Robles, Cisneros \& Guzmán, 2018). Por tanto, una posible intervención podría ser implementar estrategias didácticas innovadoras como la gamificación, el aula invertida y otras que promuevan la distensión en los estudiantes.

Sin embargo, la historia de los alumnos también puede ser un factor de influencia. Por ejemplo, se sabe que los que tienen contacto con la disciplina previamente al pregrado, ya sea mediante ejercicios, textos e incluso manipulando calculadoras, desarrollan actitudes positivas, agrado y confianza en sus habilidades para cursar la materia durante la universidad en contraste con los que nunca tuvieron dicha aproximación (Vilá \& Rubio, 2016). Por tanto, otra intervención importante tendría que proceder de la planeación curricular, pues las autoridades educativas podrían establecer a la estadística como asignatura indispensable en cualquiera de los programas de estudio previos a la licenciatura o incluso dar cursos propedéuticos en la materia previo al ingreso al pregrado.

Por otro lado, los hallazgos coinciden con la literatura en el sentido de que los alumnos utilizan a la estadística principalmente para comprender información, calcular datos y realizar investigaciones (Gil, 2003; Osorio \& Suárez, 2014; Rouquette, Suárez \& Ariza, 2014; Tapia, 2010) y de manera moderada, al final de la carrera, para la toma de decisiones (Barreto, 2012). Cabe señalar que la creencia de que la disciplina es inútil es común durante la primera etapa, pero luego desciende.

Sin embargo, llama la atención que la muestra del presente estudio utilice moderadamente la estadística en el ámbito laboral, lo cual contradice la literatura que señala el carácter imprescindible de la misma en el área económico administrativa (Jiménez, Ocegueda, García \& 
Guzmán, 2013; Liquidano, 2009; Pasten, Montiel \& Torres, 2010; Tapia, 2010). Lo anterior debe ser estudiado a profundidad, sobre todo en los alumnos de los últimos semestres, ya que el perfil de egreso de las carreras estudiadas subraya la adquisición de competencias básicas en la disciplina. Una explicación podría ser que los participantes trabajan en ámbitos ajenos a sus estudios lo cual es un tema aparte digno de explorar.

Que el porcentaje de alumnos que considera que la disciplina no le sirve disminuya entre la etapa inicial y final (ver Figura 2) podría explicarse porque, en efecto, en los primeros semestres de la carrera la mayoría aún no trabaja y tampoco hace investigación; es decir, no tienen como aplicar sus conocimientos. Una posible estrategia para este punto sería enseñar estadística con base en proyectos de tal manera que los estudiantes perciban un uso inmediato durante los primeros semestres y se motiven más con la asignatura.

Por otra parte, entre las limitaciones del presente estudio, se encuentra que el muestreo fue no aleatorio. Otra limitación fue la disparidad en la cantidad de alumnos por cada etapa de pregrado. Además, la muestra estudiada se conformó en su mayoría por mujeres; por tanto, los resultados del presente trabajo deben ser tomados como exploratorios. Ante esto, estudios posteriores deberán indagar con mayor profundidad el tema, por ejemplo, las razones por las cuales la estadística no se aplica de manera frecuente en el ámbito laboral, si existen diferencias por género, etc.

En conclusión, los estudiantes creen que la estadística es útil (principalmente en los últimos semestres de su carrera) pero también que produce tensión. Por tanto, será importante implementar estrategias para que los individuos disfruten la disciplina $\mathrm{y}$, por tanto, la utilicen con mayor frecuencia para tomar decisiones certeras en los diferentes ámbitos de su vida.

\section{Referencias}

Acosta, J., \& Mejía, D. (2017). Actitudes hacia la estadística de los estudiantes de pregrado de la universidad nacional Pedro Ruiz Gallo. Revista Científica Institucional Tzhoecoen, 9(3), 114. doi: https://doi.org/10.26495/rtzh179.323428

Aguilar, W., Lara, M., Íñiguez, C., \& Rivera, R. (2018). Perfiles de estudiantes asociados a las características de reprobación de las asignaturas de ciencias básicas en Ingeniería. Revista de la Red Iberoamericana de Pedagogía, 7(8), 129-145. https://dialnet.unirioja.es/servlet/articulo?codigo $=6523207$ 
Alonso, C. (2013). De 37\% el último índica de reprobación en la Universidad Autónoma. La Jornada. Recuperado de https://www.lja.mx/2013/10/de-37-el-ultimo-indice-dereprobacion-en-la-universidad-autonoma/

Arce, D., Andrade, E., \& Seoane, G. (1997). Comparación de escalas de respuesta para la medición de las actitudes. Psicothema, 9(3), 541-545. http://www.psicothema.com/pdf/125.pdf

Ashaari, N., Judi, H., Mohamed, H., \& Wook, M. (2010). Student's attitude towards statistics course. Procedia Social and Behavioral Sciences, 18(2011), 287-294. doi: https://doi.org/10.1016/j.sbspro.2011.05.041

Barreto, A. (2012). El progreso de la Estadística y su utilidad en la evaluación del desarrollo.

Papeles de Población, 18(73), 1-31.

http://www.scielo.org.mx/pdf/pp/v18n73/v18n73a10.pdf

Bautista, A., Morales, M., Dórame, L., \& Peralta, G. (2016). Un estudio sobre las actitudes hacia la estadística en estudiantes universitarios. Epistemus, 10(20), 48-54. http://www.epistemus.uson.mx/revistas/articulos/20-7Estadistica.pdf

Coetzee, S., \& Van der Merwe, P. (2010). Industrial psychology students' attitudes towards statistics. SA Journal of Industrial Psychology, 36(1), 1-8. doi: 10.4102/ sajip.v36i1.843

Corona, V., Reyes, S., Martínez, S., \& Rivas, C. (2016). Estrategias para la disminución de los índices de reprobación en el Instituto Tecnológico de Pachuca. Revista de Sistemas y Gestión Educativa, 3(9), 62-69.

http://www.ecorfan.org/bolivia/researchjournals/Sistemas_y_Gestion_Educativa/vol3num9 /Sistemas_y_Gestion_Educativa_V3_N9_8.pdf

Cuadras, C. (2006). La Estadística, un instrumento para el progreso de la sociedad. Boletín de Estadística e Investigación Operativa, 22(1), 29-38.

Díaz, C. (2012). Tendencias y requerimientos del mercado de trabajo en la economía del conocimiento. Estudio sobre los egresados de CUCEA. Revista de Educación Superior, 41(1), 9-30. http://publicaciones.anuies.mx/pdfs/revista/Revista161_S1A1ES.pdf

Escalante, E., Repetto, A., \& Mattinello, G. (2012). Exploración y análisis de la actitud hacia la Estadística en alumnos de psicología. Liberabit, 18(1), 15-26. http://www.scielo.org.pe/pdf/liber/v18n1/a03v18n1.pdf

Eudave, D. (2007). El aprendizaje de la estadística en estudiantes universitarios de profesiones no matemáticas. Educación Matemática, 19(2), 41-66. http://www.redalyc.org/articulo.oa?id=40519203

Gil, F. (2003). La estadística en la investigación educativa. Revista de Investigación Educativa, 21(1), 231-248. https://revistas.um.es/rie/article/view/99191/94791 
Griffith, J., Adams, L., Gu, L., Hart, C., \& Nichols, P. (2012). Students' attitudes toward statictics across the disciplines: a mixed-methods approach. Statistics Education Research Journal, 11(2), 45-56. https://iase-web.org/documents/SERJ/SERJ11(2)_Griffith.pdf

Jiménez, C., Ocegueda, C., García, I., \& Guzmán, I. (2013). Perfil del administrador de recursos humanos y desempeño organizacional en el sector industrial de H. Matamoros. Trabajo presentado en el VIII congreso para la facultad de Ingeniería Mecánica y Electrónica del 12 al 13 de septiembre en Nuevo León, México. Recuperado desde http://eprints.uanl.mx/8572/1/PERFIL\%20DEL\%20ADMINISTRADOR.pdf

Landa, M. (2015). Actitud hacia la estadística por parte de los estudiantes de Medición Psicológica de la UNAH, 2015. Revista Ciencia y Tecnología, 17, 68-78. doi: https://doi.org/10.5377/rct.v0i17.2681

Liquidano, M. (2009). Las prácticas compartidas de Administración de Recursos Humanos en PYMES de servicios de Ixtapa - Zihuatanejo. ConConciencia Tecnológica 37, 5-12. https://dialnet.unirioja.es/servlet/articulo?codigo $=6490130$

Osorio, M., \& Suárez, A. (2014). Importancia de la probabilidad y la estadística en la formación del Ingeniero. Investigación Innovación Ingeniería, 2, 26-37. doi: https://doi.org/10.24267/23462329.63

Páez, Y., Burbe, C., Mosconi, S., \& Montenegro, S. (2017). Actitudes de estudiantes hacia la estadística, antes y después de cursar la asignatura, en una escuela médica Argentina. Revista Educación en Ciencias de la Salud, 14(2), $109 \quad-114$. https://dialnet.unirioja.es/servlet/articulo?codigo $=6290864$

Pastén, C., Montiel, M., \& Torres, A. (2010). Relación de las prácticas compartidas de administración de recursos humanos y el contexto organizacional. Trabajo presentado en el XV Congreso de Contaduría, Administración e Informática para la Asociación Nacional de Facultades y Escuelas de Contaduría y Administración. http://www.redalyc.org/pdf/944/94411773002.pdf

Peña, A., Suárez, A., Sanjuán, R., Rabell, G., Gómez, M., \& Morales, I. (2015). Actitudes hacia la asignatura de Estadística en estudiantes de la Facultad de Ciencias Médicas "General Calixto García". Revista Habanera de Ciencias Médicas, 14(6), 872-883. http://scielo.sld.cu/pdf/rhcm/v14n6/rhcm16615.pdf

Pérez, G., Arango, M., \& Branch, J. (2008). El semántico diferencial como propuesta metodológica para caracterizar el liderazgo en una organización. Dyna, 75(155), 15-27. http://www.scielo.org.co/pdf/dyna/v75n155/a02v75n155.pdf

Pérez, L., Aparicio, J., Bazán, J. \& Abdounur, O. (2015). Actitudes hacia la estadística de estudiantes universitarios de Colombia. Educación Matemática, 27(3), 111-149. http://www.scielo.org.mx/scielo.php?script=sci_arttext\&pid=S1665-58262015000300111 
Petriz, M. (2015). Correlación entre actitudes hacia la Estadística y los conocimientos elementales de la disciplina en estudiantes de las licenciaturas de contaduría y administración en una universidad estatal. Recuperado desde http://congreso.investiga.fca.unam.mx/docs/xx/docs/8.04.pdf

Robles, S., Cisneros, L., \& Guzmán, C. (2018). Actitudes hacia las matemáticas de estudiantes universitarios. El caso del Centro Universitario de Ciencias Económico Administrativas de la Universidad de Guadalajara. Revista de Educación y Desarrollo, 47, 71-80. http://www.cucs.udg.mx/revistas/edu_desarrollo/anteriores/48/48_Robles.pdf

Rodríguez, N. (2011). Actitudes de los estudiantes universitarios hacia la Estadística. Interdisciplinaria, 28(2), 199-205.

Rosli, M., \& Maat, S. (2017). Attitude towards statistics and performance among post-graduate students. Recuperado desde https://aip.scitation.org/doi/pdf/10.1063/1.4983881?class=pdf

Rouquette, A. Suárez, A., \& Ariza, E. (2014). Relevancia de la formación estadística en la universidad. La importancia de encontrarles sentido a las matemáticas. Reencuentro, 69, 3745. http://www.redalyc.org/pdf/340/34031038005.pdf

Ruiz, C. (2015). Actitudes hacia la estadística de los alumnos del grado en pedagogía, educación social, y maestro de educación infantil y maestro de educación primaria de la UCM. Educación XX1, 18(2), 351-374.

Salazar, M., \& Acón, S. (2017). Actitudes de estudiantes universitarios hacia la estadística como materia de estudio y herramienta para el análisis y comprensión de situaciones cotidianas. Trabajo presentado en el V Encuentro de Enseñanza de la Matemática UNED 2017. San José, CR. Resumen recuperado de https://www.uned.ac.cr/ecen/encuentros/2017/vencuentro/archivos/Ponencias/4.\%20Did\% C3\%A1cticas\%20especificas\%20en\%20la\%20educaci\%C3\%B3n\%20matem\%C3\%A1tica /Actitudes\%20estudiantes\%20universitarios\%20hacia\%20estad\%C3\%ADstica.pdf

Salkind, N. (1999). Métodos de investigación. $3^{\mathrm{a} e d . ~ M e ́ x i c o: ~ P r e n t i c e ~ H a l l . ~}$

Santabarbara, J., \& López, R. (2019). Actitudes hacia la estadística en residentes de MediCuba que cursan un posgrado en investigación. Revista de la Fundación Médica, 22(2), 79-83. https://dialnet.unirioja.es/servlet/articulo?codigo $=6896427$

Sesé, A., Jiménez, R., Montaño, J., \& Palmer, A. (2015). ¿Pueden las actitudes hacia la estadística y la ansiedad estadística explicar el rendimiento de los estudiantes? Revista de Psicodidáctica, 20(2), 285-304. doi: 10.1387/RevPsicodidact.13080.

Stanisavljevic, D., Trajkovic, G., Marinkovic, J., Bukumiric, Z., Cirkovic, A., \& Milic, N. (2014). Assessing attitudes towards statistics among medical students: psychometric properties of the Serbian version of the survey of attitudes towards statistics (SATS). PLoS ONE 9(11), 1-7. doi:10.1371/journal.pone.0112567. 
Vilá, R., \& Rubio, M. (2016). Actitudes hacia la Estadística en el alumnado del grado de Pedagogía de la Universidad de Barcelona. Revista de Docencia Universitaria, 14(1), 131-149. doi: $\underline{10.4995 / \mathrm{redu} .2016 .5766}$ 